\title{
THE
}

\section{Sleep Duration Mediates the Relationship Between Health Behavior Patterns and Obesity}

Jill L. Kaar

Sarah J. Schmiege

Maya Vadiveloo

University of Rhode Island, maya_vadiveloo@uri.edu

Stacey L. Simon

Alison Tovar

University of Rhode Island, alison_tovar@uri.edu

Follow this and additional works at: https://digitalcommons.uri.edu/nfs_facpubs

The University of Rhode Island Faculty have made this article openly available.

Please let us know how Open Access to this research benefits you.

This is a pre-publication author manuscript of the final, published article.

Terms of Use

This article is made available under the terms and conditions applicable towards Open Access Policy Articles, as set forth in our Terms of Use.

\section{Citation/Publisher Attribution}

Kaar, J. L., Schmiege, S. J., Vadiveloo, M., Simon, S. L., \& Tovar, A. (2018). Sleep Duration Mediates the Relationship Between Health Behavior Patterns and Obesity. Sleep Health, 4(5), 442-447. doi: 10.1016/ j.sleh.2018.07.004

Available at: https://doi.org/10.1016/j.sleh.2018.07.004

This Article is brought to you for free and open access by the Nutrition and Food Sciences at DigitalCommons@URI. It has been accepted for inclusion in Nutrition and Food Sciences Faculty Publications by an authorized administrator of DigitalCommons@URI. For more information, please contact digitalcommonsgroup@uri.edu. 
Sleep Duration Mediates the Relationship Between Health Behavior Patterns and Obesity

Authors: Jill L Kaar $\mathrm{PhD}^{1}$, Sarah J Schmiege $\mathrm{PhD}^{2}$, Maya Vadiveloo PhD RD ${ }^{3}$, Stacey L Simon $\mathrm{PhD}^{4}$, and Alison Tovar $\mathrm{PhD}^{3}$

${ }^{1}$ Department of Pediatrics, Division of Pediatric Endocrinology, Children's Hospital Colorado and University of Colorado Anschutz Medical Campus, Aurora, Colorado

${ }^{2}$ Department of Biostatistics and Informatics, Colorado School of Public Health, University of Colorado Anschutz Medical Campus, Aurora, Colorado

${ }^{3}$ Department of Nutrition and Food Sciences, University of Rhode Island, Kingston, Rhode Island

${ }^{4}$ Department of Pediatrics, Division of Pulmonology, Children's Hospital Colorado and University of Colorado Anschutz Medical Campus, Aurora, Colorado

Running Title: sleep, health behavior patterns, and obesity

Corresponding Author: Jill Landsbaugh Kaar, PhD, Children's Hospital Colorado, Section of Endocrinology, 13123 East $16^{\text {th }}$ Avenue, Box 265, Aurora, CO 80045; Phone: (720) 777-3571;

Email: Jill.Kaar@ucdenver.edu 
Abstract

Objective: To examine associations between health behavior patterns and childhood obesity, and the mediating effect of sleep duration.

Design: Population-based survey.

Participants: Secondary analysis of data from the Infant Feeding Practices Study (age 6 years, $\mathrm{n}=1073$ ).

Measurements: Mothers self-reported their child's health behaviors including physical activity (PA), screen time, sleep duration, and diet. Latent class analysis determined the child's patterns based on health behaviors. Sleep was examined as a mediator between the class membership variable and $\% \mathrm{BMI}_{\mathrm{p} 95}$.

Results: A 3-class model fit the data best, with classes labeled as "Poorest eaters"(low fruit/vegetable consumption, high fast food), "Healthy"(low screen time, highest fruit/vegetable consumption) and "Active, super-eaters, highest screen time"(highest PA and screen time, ate the most). "Poorest eaters" had an increased \%BMI $95(\beta=4.11, \mathrm{p}=0.006)$ relative to the "Healthy" class. The "Poorest eaters" and "Active, super-eaters, highest screen time" classes had shorter sleep duration ( $\beta=-0.51, \mathrm{p}<0.001 ; \beta=-0.38, \mathrm{p}<0.001$; respectively) relative to the "Healthy" class. Independent of class membership, each additional hour of sleep was associated with a $\% \mathrm{BMI}_{\mathrm{p} 95}$ that was 2.93 units lower $(\mathrm{p}<0.001)$.

Conclusions: Our results indiciate that health behavior patterns mediated by sleep duration may influence a child's \% $\mathrm{BMI}_{\mathrm{p} 95}$. The bi-directionality of the relationship beteen health behaviors and sleep remains unclear. Our findings suggest the importance of a constellation of health behaviors on childhood obesity. Interventions should include a multitude of health behaviors and consider the possibility that improving diet and activity behaviors may facilitate improved sleep and lowered obesity risk among children.

Keywords: sleep, activity, pediatrics, obesity, diet 
Introduction

Pediatric obesity continues to be a public health crisis with one in three children classified as overweight or obese. ${ }^{1}$ Given that children with obesity are more likely to become adults with obesity and its related adverse consquences, ${ }^{2}$ there is an urgent need to better understand the pathways by which modifiable health behaviors influence obesity risk, as well as potential mediators of this pathway, such as sleep duration. Sleep problems are among the most common parental complaints to pediatricians, ${ }^{3}$ and preschool-age children are more likely to be diagnosed with a sleep disorder than any other age group. ${ }^{4}$ Recent evidence suggests that insufficient sleep is associated with greater risk for obesity. ${ }^{5}$ However, how sleep interacts with health behaviors to influence obesity risk obesity risk remains unclear. Insufficient sleep may negatively impact health behaviors (e.g., diet, physical activity, screen time) which in turn could lead to increased obesity risk. In a study of over 1,000 children aged 7 years, children who slept for longer durations were found to have a healthier diet and lower BMI z-score. ${ }^{6}$ Similar findings in a cohort Canadian children found that parent reported longer sleep duration was associated with a better quality of diet as well as higher levels of physical activity. ${ }^{7}$ Alternatively, it is possible that unhealthy behaviors could result in poorer sleep which in turn may influence obesity risk. The goal of this paper is to test the latter pathway to answer the question of whether sleep mediates the relationship between health behavior patterns and obesity risk in children. By better understanding this potential pathway, effective interventions to prevent and treat obesity early in life can be designed.

Health behaviors such as diet and activity have shown bivariate associations with sleep duration. Certain dietary behaviors, for example habitual snacking throughout the day and high caffeine consumption, have been associated with shorter sleep duration; conversely certain fruits and dairy products have been associated with improved sleep. ${ }^{8}$ Physical activity (PA), particularly 
moderate-vigorous PA, has been associated with improved sleep. ${ }^{9}$ In contrast, screen time use has been consistently associated with shortened sleep duration. ${ }^{10}$ Not only have health behaviors been associated with sleep duration, but sleep duration has been linked to obesity risk independent of these health behaviors..$^{11-13}$

The underlying mechanism driving the sleep-obesity association, independent of diet and activity behaviors, appears to be through changes in metabolism. For example, in tightly controlled, laboratory-based sleep restriction studies among adults, shortened sleep duration had negative effects on hormones (i.e., leptin, glucose, insulin), which induced increases in weight. ${ }^{14}$ Other adult studies also found significant decreases in body temperature following sleep restriction which could eventually lead to decreases in basal metabolic rate, thus impacting weight. ${ }^{14}$ In pediatric populations, negative hormonal changes have occurred in individuals with circadian rhythm misalignment and sleep restriction (i.e., late bed times, early wake times). ${ }^{15,16}$ However, whether insufficient sleep influences metabolism directly, or indirectly, through changes in diet and physical activity, is unknown, particularly in young children.

In understanding the pathway (Figure 1) by which sleep influences the association between health behaviors and obesity, it is important to capture health behaviors as they pattern together. For example, although there are a number of studies exploring the unique contribution of sleep, diet, screen time and physical activity behaviors individually on obesity, it remains unclear how these health behaviors together impact risk for obesity. To date, an underlying assumption has been that obesity risk is the same for a given "dose" of an exposure. ${ }^{17}$ For example, while high sugarsweetened beverage intake may increase obesity risk controlling for confounders, it is plausible that very active children may be protected against the effects of energy-dense nutrient poor quality diets whereas children with poor sleep duration or quality may respond even more strongly to 
dietary risk factors. Thus it is critical to better understand how different health behavior clusters ultimately influence childhood obesity risk.

Health behaviors co-occur, and obese children most often report having two or more eating and activity risk behaviors. ${ }^{18}$ It is possible that these health behaviors patterns may better predict obesity risk rather than focusing on single behaviors; unfortunately, few studies have examined behavior patterns, ${ }^{19-22}$ only two of these in childhood. ${ }^{19,20}$ Furthermore, no studies to our knowledge have used these patterns in understanding the pathway by which sleep mediates the association between health behaviors and obesity. Therefore, the objective of this analysis is to examine the mediating effect of sleep duration in the relationship between health behavior patterns and childhood obesity. We hypothesize that the association between health behavior patterns and obesity risk is at least partially mediated by sleep duration in children.

\section{Methods}

Participants

This was a secondary analysis using the nationally distributed Infant Feeding Practice Survey II (IFPS II) and Year 6 Follow-Up Study (Y6FU). ${ }^{23,24}$ The IFPS II is a prospective, longitudinal cohort, funded by the Centers for Disease Control and Food and Drug Administration (CDC), that followed women in late pregnancy and their children through age 6 years. The sample for Y6FU was a subset of those who participated in IFPS II. Mother who completed at least the first postnatal questionnaire for IFPS II were contacted to participant in Y6FU ( $n=2958$ contacted). A total of 1542 mothers completed the mailed questionnaires.

Measurements

Mailed questionnaires were completed by mothers. The 1-month Harvard Children's Nutrition 
Food Frequency Questionnaire assessed the child's diet, and a study questionnaire queried their child's activity, screen time, and sleep behaviors. ${ }^{24}$ These questionnaires were developed using previously published national surveys and pilot tested to ensure content validity as well as appropriate variation and range of responses. PA was assessed by asking "in a typical week, how many days is your 6-year old physically active for a total of at least 60 minutes per day". Screen time was assessed by asking "on average, about how many hours per day does your 6-year-old play video games and watch TV programs or videos (do not count school or homework time)". Sleep was assessed with the question "Over the past month, how many hours did your 6-year-old usually sleep each night on weekdays". Mothers were also asked to measure and self-report their child's height and weight (Y6FU) when the children were 6 years old. These data were used to calculate the $\mathrm{BMI}$ as $\%$ of the $95^{\text {th }}$ percentile $\left(\% \mathrm{BMI}_{\mathrm{p} 95}\right)$ using the CDC SAS program for growth charts. ${ }^{25}$ Social-demographic data for the family was also self-reported by the mothers. Complete information regarding this study has been previously published. ${ }^{23,24}$ Statistical Analysis

Our analysis plan consisted of three steps. First, we created dichotomous versions of our seven health behavior indicator variables using the Youden index. Next we carried out a latent class analysis (LCA) to identify health behavior patterns using these seven indicators. Lastly, we conducted a series of regression models to examine sleep duration as a mediator of the association of health behavior patterns and obesity.

The seven health behavior indicator variables were constructed using the Youden index. By utilizing a data driven approach, the Youden index calculates the amount of a given behavior that is associated with the outcome, obesity. The threshold for each variable was chosen as the value which maximized the $J$ statistic (sensitivity + specificity -1 ) from a linear regression with 
obesity ( $\left.>\% \mathrm{BMI}_{\mathrm{p} 95}\right)$ as outcome and the specified indicator variable. The following variables were constructed; high physical activity (>60 min/day, $5 \mathrm{~d} / \mathrm{wk}$ ), high screen time (>100 min/day), high breakfast consumption ( $>5 \mathrm{~d} / \mathrm{wk})$, high fruit consumption $(1+/ \mathrm{d})$, high vegetable consumption $(1+/ \mathrm{d})$, high fast food consumption $(1+/ \mathrm{wk})$, and high sugar sweetened beverages $(1+/ \mathrm{d})$. These seven health behaviors were selected as they tend to be targets of childhood obesity treatment and prevention programs. Further a distinction of "high" was given to each behavior in order to guide interventionists in usable targets when developing such programs.

Next, we conducted latent class analysis (LCA) to identify children's health behavior patterns. LCA is a commonly used approach to identify subpopulations (i.e., latent classes) by shared item response patterns. Using this approach, patterns (i.e., classes) of individuals are expected to be similar to one another with regards to the variables of interest, but different from individuals in other patterns. LCA was conducted using Mplus Version 7.1 to identify health behavior patterns using the above seven indicators of health behaviors.

For our LCA we started with a 1-class model and increased the number of classes until the best-fitting model was observed. Following current recommendations, we reported a constellation of various types of fit indices to offset advantages/limitations of any one index. ${ }^{26}$ Relative fit indices based on information-heuristic criteria included Akaike's Information Criterion (AIC), the Bayesian Information Criterion (BIC), Consistent Akaike's Information Criterion (CAIC), and the Approximate Weight of Evidence Criterion (AWE). Additional fit statistics included the LoMendell-Rubin likelihood ratio test (LMR-LRT), the Bayes Factor, and the Correct Model Probability (cmP). The LMR-LRT compares the k-class model under consideration to a k-1 class, where a significant $\mathrm{p}$-value indicates that the estimated model fits the data significantly better than a model with one fewer class. ${ }^{27}$ The Bayes Factor compares a k-class model to a $\mathrm{k}+1$ class model 
and assesses which of the two models is more likely to be the true model, assuming one of them is true (higher is better). The $\mathrm{cmP}$ assesses the probability of a given model being correct relative to a set of alternative models (i.e., all other models under consideration). The best fitting model was chosen based on the collective information provided by model fit indices (see Table 1). Specifically, we primary utilized the BIC and the LMR-LRT in choosing a best fitting model, and relied on additional fit indices as converging evidence for that model. ${ }^{28}$

Once a final model was chosen, each participant was assigned most likely class membership, resulting in each participant being assigned membership to a categorical observed variable representative of a health behavior pattern. Using this categorical variable, a series of regression models were estimated to address our hypothesis that sleep (hours per night) mediates the association between class membership and $\% \mathrm{BMI}_{\mathrm{p} 95}$. Model 1 examined the effect of class membership on $\% \mathrm{BMI}_{\mathrm{p} 95}$; model 2 examined the effect of class membership on sleep (hours); and model 3 examined the effect of sleep (hours) on $\% \mathrm{BMI}_{\mathrm{p} 95}$ while adjusting for class membership. This "classify/analyze" approach of generating an observed variable for most likely class membership and using that variable as input data in testing the regression relationships does not address classification uncertainty; therefore, sensitivity analysis confirmed model relationships under current best practices for examining the relationship of class membership to distal outcomes while accounting for classification uncertainty, and model conclusions were unchanged. ${ }^{29}$ Due to the homogeneity of the cohort's race and ethnicity, we did not address those variables further in the analyses; we did include gender as a covariate in the regression analyses, but model results were unchanged so we report the unadjusted results for simplicity.

\section{Results}

Our complete cases for this analysis consisted of 1073 six-year-old children. Children without 
complete data relevant for our analysis were excluded ( $n=469)$; missing due to sex $(n=2)$, height/weight data $(n=329)$, race $(n=102)$, ethnicity $(n=5)$, and income $(n=31)$. There were no significant differences for SES indicators between the full cohort $(n=1542)$ and our analysis sample $(\mathrm{n}=1073)$. The large number of missing height/weight data is thought to be attributed to additional burden of collecting this data as mothers were sent a measuring tape and instructed on how to measure the child's height and weight.

Mothers in the cohort were $89 \%$ White and $25 \%$ reported a household income below $\$ 39,000$ per year. Of the children, $49 \%$ were female and $13 \%$ of the children classified as obese (BMI above the $95^{\text {th }}$ percentile). Further description of the cohort by latent class membership is provided in Table 2 .

We estimated between 1-5 class models, using multiple random perturbations of start values for each model. All models converged, there were no negative indications with regard to model identification, and the log likelihood value was replicated for all solutions. The smallest class size in the 5-class solution was $\mathrm{n}=35(3.26 \%)$, so we did not estimate beyond five classes. Model fit indices, shown in Table 1, supported the 3-class solution. As a measure of absolute fit, the likelihood ratio $\chi^{2}$ test first becomes non-significant (good-fitting) under the 3-class solution. The AIC, BIC and CAIC supported the 3-class model; while the AWE supported a 2-class model, this index tends to under-extract classes and therefore provides a lower bound estimate for class enumeration. The LMR-LRT $(\mathrm{p}=0.13)$, Bayes Factor $(>10)$, and the $\mathrm{cmP}(>0.99)$ all supported the 3-class solution. The entropy value of the 3-class model was .58, with average posterior class probabilities of $0.84,0.77$, and 0.80 across the 3 classes, respectively.

Based on measures of absolute and relative fit, we proceeded to interpret the 3-class model results. Figure 1 depicts the model-estimated item response probabilities for each of the 7 
indicators of the 3 -class solution. The first class $(\mathrm{n}=233,22 \%)$ was homogeneous with regard to low intake of fruits and vegetables and high fast food intake; we therefore labeled this class "Poorest eaters." Class $2(\mathrm{n}=399,37 \%)$ was homogeneous with respect to low rates of screen time and sugar-sweetened beverages and high rates of consuming fruits and vegetables, eating breakfast, and engaging in physical activity; we labeled this as the "Healthy" class. Class 3 $(n=441,41 \%)$ was homogeneous with high rates of physical activity, and fruit and vegetable intake but also high fast food consumption and the highest screen time of all three classes; we labeled this class "Active, super-eaters, highest screen time". In summary, the 3-class model was supported as the best fitting model in relative and absolute terms and the observed class structure was theoretically interpretable.

Three regression models were estimated to test sleep duration as a mediator between class membership and $\% \mathrm{BMI}_{\mathrm{p} 95}$ (Table 3). First the effect of class membership on $\% \mathrm{BMI}_{\mathrm{p} 95}$ was examined. Compared to the "Healthy" class, the "Poorest eaters" class had an increased $\% \mathrm{BMI}_{\mathrm{p} 95}$ of $4.11(\mathrm{p}=0.006)$. The next model examined the effect of class membership on sleep (hours). Compared to the "Healthy" class, both other classes had significantly shorter amounts of sleep ( $\beta$ $=-0.51, \mathrm{p}<0.001$ for "Poorest eaters"; $\beta-0.38, \mathrm{p}<0.001$ for "Active, super-eaters, highest screen time"). In the final model, the effect of sleep (hours) on BMI was examined adjusting for class membership. Independent of class membership, each additional hour of sleep was associated with a $\% \mathrm{BMI}_{\mathrm{p} 95}$ that was 2.93 units lower $(\mathrm{p}<0.001)$ and class membership was no longer significantly associated with $\% \mathrm{BMI}_{\mathrm{p} 95}$. Thus, the effect of class variable on $\% \mathrm{BMI}_{\mathrm{p} 95}$ appears to be fully mediated by sleep.

\section{Discussion}


The goal of this study was to explore the mediating role of sleep duration in the pathway between modifiable health behavior patterns and risk of obesity. We found that children with health behavior patterns consisting of low fruit and vegetable intake and high fast food consumption ("Poorest Eaters") had higher \%BMI ${ }_{p 55}$ compared to children with "Healthy" patterns. The "Poorest Eaters" and children classified as "Active, super-eaters, highest screen time" also had significantly reduced sleep duration compared to children classified with having a "Healthy" pattern. Finally, we found that sleep duration mediated the association of health behavior patterns and a child's $\% \mathrm{BMI}_{\mathrm{p} 95}$. Pediatric lifestyle management interventions should include a multitude of health behaviors and consider the possibility that improving diet and activity behaviors may facilitate improved sleep and obesity risk among children.

Our finding that poor health behavior patterns are associated with obesity risk are consistent with prior work. For example, two similar studies conducted in early childhood (4-6 years) found that unhealthy behaviors, in particular high screen time, are associated with obesity. ${ }^{19,20}$ Our work, however, extends findings from the previous literature by implicating sleep duration in the pathway linking health behaviors with obesity. Although prior studies have not included sleep duration, they have examined individual behaviors (i.e., diet) and sleep duration in relation to obesity. Parent-reported sleep duration has been linked to diet and activity behaviors in a cohort of 10-11 year old Candian children. A significant increase in both self-reported diet quality and physical activity were shown for every additional hour of sleep duration. ${ }^{7}$ Further, longer sleep duration was associated with decreased odds of being classified as overweight or obese. The Project Viva cohort found that chronic insufficient sleep (defined as short sleep duration from infancy to age 7) was associated with poor diet quality. ${ }^{6}$ However, after further testing, poor diet quality did not explain the association between sleep and childhood obesity. In the same cohort, 
using data from the 7-year visit only, children with the highest sleep scores (defined as mean sleep duration) had the lowest BMI z-scores and higher self-reported diet quality scores. In a multiethnic cohort of 2-4 year old children, obese children with poor sleep duration (defined as less than 11 hours of sleep per night), had significantly higher intakes $(\mathrm{g} / \mathrm{d})$ of fat and saturated fat as well as a higher percentage of their overall dietary intake (\% of kcal) from fat and significantly lower percent calories from carbohydrate during the weekdays. ${ }^{30}$

We found that the relationship between the health behaviors and obesity was no longer significant in our model 3 with the inclusion of sleep duration, suggesting that sleep may be an important mechanism linking health behaviors to obesity. Although these findings suggest that sleep mediates the relationship between health behaviors and obesity it is also possible that lack of sleep can have a negative impact on health behaviors which in turn impact obesity. Research has been conducted with the hypothesis that children with shorter sleep duration engage more often in obesity-related behaviors including lower levels of physical activity, higher screen time, lower consumption of fruits and vegetables and higher consumption of sugar sweetened beverages. ${ }^{31-}$ ${ }^{33}$ Future work is needed to disentangle this relationship further to better understand where the cycle starts (Figure 1).

Moving forward, future studies should consider examining this question at a more mechanistic level to better understand how the cycle influences metabolism and specific hormones associated with poor health behaviors and sleep. Unfortunately, this cohort did not collect hormonal or metabolic data in this young sample, but our findings highlight the need for future studies to assess these factors when attempting to elucidate the mechanism behind the association between sleep, health behaviors, and obesity. Further, there may be other factors outside of diet and activity behaviors that impact sleep duration; including family routines and/or chaos..$^{34,35}$ 
Recent evidence suggests that screen time in particular may play an important role on sleep through the effect of bright light in suppressing sleep promoting melatonin and delaying circadian rhythms, ${ }^{36-38}$ although a causal association has not been confirmed. ${ }^{10,39}$ Given that screens are becoming more ubiquitous in children's everyday life, future research should continue to tease out the impact of this behavior on sleep and obesity risk. Early evidence suggests that high family chaos plays a significant role in child sleep problems. ${ }^{35}$ These and other health behaviors which were not able to be included in our analysis may also explain our findings and should be included in future research.

The results of this study provide preliminary evidence that specific health behavior patterns early in life are associated with obesity in children as young as six years. We utilized a novel approach on early health behavior patterns including diet, physical activity, and screen time in relation to indicators of obesity. Further, we were able to examine how multiple health behaviors may pattern together and then test how this association was influenced by sleep duration. To our knowledge, this is the first study to conduct this analysis. Our approach of strong quantitative analysis permitted us to examine multiple behaviors simultaneously compared to other approaches which focus only on one health behavior at a time while holding others constant. By exploring patterns of relevant obesity-related health behaviors and how they interrelate to influence weight status, our results can more efficiently guide future obesity prevention and treatment strategies by developing more effective and targeted interventions.

Our findings should be interpreted cautiously and may not be representative of all young children. The Infant Feeding Practices II cohort consists of predominantly White maternal-child dyads with higher incomes. Similar to other studies published to date, the measures of health behaviors, child height and weight, and sleep were obtained by parent-report and not verified by 
medical records or objective measurements. The gold standard for sleep measurement is polysomnography, and actigraphy is able to objectively capture typical sleep across a longer duration in the home environment. Similarly, accelerometers can be used to objectively measure physical activity. Future research should evaluate relationships using these objective measures of health and health behaviors. Moreover, the current study only examined sleep duration yet sleep is a multidimensional construct and thus other variables such as sleep quality and variability should also be considered. Another limitation is the use of dichotomous health indicators for the pattern analysis. We utilized dichotomous cut-points for each of the health behaviors to improve public health relevance after initial modeling using continuous indicators resulted in difficulty with model estimation/interpretation, but recognize drawkbacks of categorizing continuous measures. Finally, because these analyses used cross-sectional data, the directionality of these associations cannot be determined. However, given the robust research linking childhood health behaviors and childhood obesity risk, reverse causality is unlikely. This study also has a number of strengths including the large sample size, prospective design, use of extensively tested survey questions, and wealth of data collected related to childhood obesity risk. Further work should be conducted to replicate these findings in more diverse, longitudinal cohorts and if possible, with more objective measures of key study variables such as physical activity and sleep.

\section{Conclusion}

In conclusion, we observed sleep duration as a potential pathway linking health behavior patterns and obesity in children. Future research should continue to explore the metabolic and behavioral pathways by which this occurs. In addition, future interventions may want to explore the improvement of health behavior patterns which in turn may improve a child's sleep duration and subsequently, BMI. 


\section{References}

1. Skinner AC, Ravanbakht SN, Skelton JA, Perrin EM, Armstrong SC. Prevalence of obesity and severe obesity in US children, 1999-2016. Pediatrics. 2018.

2. Gurnani M, Birken C, Hamilton J. Childhood obesity: causes, consequences, and management. Pediatric clinics of North America. 2015;62(4):821-840.

3. Armstrong KL, Quinn RA, Dadds MR. The sleep patterns of normal children. The Medical Journal of Australia. 1994;161(3):202-206.

4. Meltzer LJ, Johnson C, Crosette J, Ramos M, Mindell JA. Prevalence of diagnosed sleep disorders in pediatric primary care practices. Pediatrics. 2010;125(6):e1410-1418.

5. Miller MA, Kruisbrink M, Wallace J, Ji C, Cappuccio FP. Sleep duration and incidence of obesity in infants, children and adolescents: A systematic review and meta-analysis of prospective studies. Sleep. 2018; 41(4).

6. Cespedes EM, Hu FB, Redline S, et al. Chronic insufficient sleep and diet quality: Contributors to childhood obesity. Obesity (Silver Spring, Md). 2016;24(1):184-190.

7. Khan MK, Chu YL, Kirk SF, PJ V. Are sleep duration and sleep quality associated with diet quality, physical activity, and body weight status? A population-based study of Canadian children. Can J Public Health. 2015;106(5):e277-282.

8. Peuhkuri K, Sihvola N, Korpela R. Diet promotes sleep duration and quality. Nutrition Research (New York, NY). 2012;32(5):309-319.

9. Chennaoui M, Arnal PJ, Sauvet F, Leger D. Sleep and exercise: a reciprocal issue? Sleep Medicine Reviews. 2015;20:59-72.

10. Hale L, Guan S. Screen time and sleep among school-aged children and adolescents: a systematic literature review. Sleep Medicine Reviews. 2015;21:50-58.

11. Beccuti G, Pannain S. Sleep and obesity. Current Opinion in Clinical Nutrition and Metabolic Care. 2011;14(4):402-412.

12. Bell JF, Zimmerman FJ. Shortened nighttime sleep duration in early life and subsequent childhood obesity. Archives of Pediatrics \& Adolescent Medicine. 2010;164(9):840-845.

13. Nedeltcheva AV, Kilkus JM, Imperial J, Schoeller DA, Penev PD. Insufficient sleep undermines dietary efforts to reduce adiposity. Ann Intern Med. 2010;153(7):435-441.

14. Patel SR, Hu FB. Short sleep duration and weight gain: a systematic review. Obesity (Silver Spring, Md). 2008;16(3):643-653.

15. Owens JA, Weiss MR. Insufficient sleep in adolescents: causes and consequences. Minerva Pediatrica. 2017.

16. Hart CN, Carskadon MA, Considine RV, et al. Changes in children's sleep duration on food intake, weight, and leptin. Pediatrics. 2013;132(6):e1473-1480.

17. Qi L. Personalized nutrition and obesity. Annals of Medicine. 2014;46(5):247-252. 
18. Sanchez A, Norman GJ, Sallis JF, Calfas KJ, Rock C, Patrick K. Patterns and correlates of multiple risk behaviors in overweight women. Prev Med. 2008;46(3):196-202.

19. Gubbels JS, Kremers SP, Stafleu A, Goldbohm RA, de Vries NK, Thijs C. Clustering of energy balance-related behaviors in 5-year-old children: lifestyle patterns and their longitudinal association with weight status development in early childhood. Int J Behav Nutr Phys Act. 2012;9:77.

20. Lioret S, Touvier M, Lafay L, Volatier JL, Maire B. Dietary and physical activity patterns in French children are related to overweight and socioeconomic status. J Nutr. 2008;138(1):101-107.

21. Schmiege SJ, Gance-Cleveland B, Gilbert L, Aldrich H, Gilbert KC, Barton A. Identifying patterns of obesity risk behavior to improve pediatric primary care. Journal for Specialists in Pediatric Nursing : JSPN. 2015.

22. Leech RM, McNaughton SA, Timperio A. The clustering of diet, physical activity and sedentary behavior in children and adolescents: a review. Int J Behav Nutr Phys Act. 2014;11:4.

23. Fein SB, Labiner-Wolfe J, Shealy KR, Li R, Chen J, Grummer-Strawn LM. Infant Feeding Practices Study II: study methods. Pediatrics. 2008;122 Suppl 2:S28-35.

24. Fein SB, Li R, Chen J, Scanlon KS, Grummer-Strawn LM. Methods for the year 6 followup study of children in the Infant Feeding Practices Study II. Pediatrics. 2014;134 Suppl 1:S4-s12.

25. Kuczmarski RJ, Ogden CL, Guo SS. 2000 CDC growth charts for the United States: methods and development. In: National Center for Health Statistics, ed. Vol 11 (246): Vital Health Stat; 2002.

26. Masyn K. Latent class analysis and finite mixture modeling. Vol 2. New York, NY: Oxford University Press; 2013.

27. Nylund KL, Asparouhov T, Muthén BO. Deciding on the number of classes in latent class analysis and growth mixture modeling: A Monte Carlo simulation study. Structural Equation Modeling: A Multidisciplinary Journal. 2007;14(4):535-569.

28. Nylund KL, Asparouhov T, Muthén BO. Deciding on the number of classes in latent class analysis and growth mixture modeling: A Monte Carlo simulation study, . Structural Equation Modeling: A Multidisciplinary Journal. 2007;14(4):535-569.

29. Asparouhov T, Muthén B. Auxiliary variables in mixture modeling: Using the $\mathrm{BCH}$ method in Mplus to estimate a distal outcome model and an arbitrary second model. Mplus Web Notes 2014; No. 21.

30. Petrov ME, Vander Wyst KB, Whisner CM, et al. Relationship of sleep duration and regularity with dietary intake among preschool-aged children with obesity from lowincome families. Journal of Developmental and Behavioral Pediatrics : JDBP. 2017;38(2):120-128.

31. Hager ER, Calamaro CJ, Bentley LM, Hurley KM, Wang Y, Black MM. Nighttime sleep duration and sleep behaviors among toddlers from low-income families: Associations with obesogenic behaviors and obesity and the role of parenting. Childhood Obesity (Print). 2016;12(5):392-400.

32. Kjeldsen JS, Hjorth MF, Andersen R, et al. Short sleep duration and large variability in sleep duration are independently associated with dietary risk factors for obesity in Danish school children. International Journal of Obesity (2005). 2014;38(1):32-39. 
33. Golley RK, Maher CA, Matricciani L, Olds TS. Sleep duration or bedtime? Exploring the association between sleep timing behaviour, diet and BMI in children and adolescents. International Journal of Obesity (2005). 2013;37(4):546-551.

34. Falbe J, Davison KK, Franckle RL, et al. Sleep duration, restfulness, and screens in the sleep environment. Pediatrics. 2015;135(2):e367-375.

35. Boles RE, Halbower AC, Daniels S, Gunnarsdottir T, Whitesell N, Johnson SL. Family chaos and child functioning in relation to sleep problems among children at risk for obesity. Behavioral Sleep Medicine. 2017;15(2):114-128.

36. Higuchi S, Motohashi Y, Liu Y, Maeda A. Effects of playing a computer game using a bright display on presleep physiological variables, sleep latency, slow wave sleep and REM sleep. J Sleep Res. 2005;14(3):267-273.

37. Gooley JJ, Chamberlain K, Smith KA, et al. Exposure to room light before bedtime suppresses melatonin onset and shortens melatonin duration in humans. The Journal of Clinical Endocrinology and Metabolism. 2011;96(3):E463-472.

38. Wood B, Rea MS, Plitnick B, Figueiro MG. Light level and duration of exposure determine the impact of self-luminous tablets on melatonin suppression. Applied Ergonomics. 2013;44(2):237-240.

39. Domingues-Montanari S. Clinical and psychological effects of excessive screen time on children. J Paediatr Child Health. 2017;53(4):333-338.

Figure 1. Cyclic relationship of poor health behaviors worsening sleep Figure 2. Model-estimated item response probabilities by class. 
Table 1. Model fit indices for sample ( $\mathrm{n}=1073)$.

\begin{tabular}{|c|c|c|c|c|c|c|c|c|c|c|}
\hline Model & LL & npar & $\begin{array}{l}\text { Adjusted } \chi^{2} \text { LR (df), p- } \\
\text { value }\end{array}$ & AIC & $\mathrm{BIC}$ & CAIC & AWE & $\begin{array}{c}\text { Adjusted } \\
\text { LMR-LRT } \\
\text { p value }\end{array}$ & $\mathrm{BF}$ & $\mathrm{cmP}$ \\
\hline 1-class & -4156.50 & 7 & $440.86(120),<.001$ & 8327 & 8362 & 8369 & 8432 & $<.001$ & $<.01$ & $<.01$ \\
\hline 2-class & -4027.69 & 15 & $183.24(112),<.001$ & 8085 & 8160 & 8175 & 8310 & $<.001$ & $<.01$ & $<.01$ \\
\hline 3-class & -3987.83 & 23 & $103.53(104), 0.49$ & 8022 & 8136 & 8159 & 8366 & 0.13 & $>10$ & $>.99$ \\
\hline 4-class & -3980.09 & 31 & 217.97 (220), 0.71 & 8022 & 8177 & 8208 & 8486 & 0.06 & $>10$ & $<.01$ \\
\hline 5-class & -3972.65 & 39 & 110.44 (193), 0.87 & 8023 & 8217 & 8256 & 8607 & -- & -- & $<.01$ \\
\hline
\end{tabular}

$\mathrm{LL}=\log$ likelihood value; npar $=$ number of parameters estimated; LR=Likelihood ratio; AIC = Akaike's Information Criterion; BIC $=$ Bayesian Information Criterion; CAIC = Consistent Akaike's Information Criterion; AWE = Approximate Weight of Evidence Criterion; LMR-LRT = Lo Mendall Rubin Likelihood Ratio Test; BF = Bayes Factor; cmP = Correct Model Probability. 
Table 2. Description of cohort

\begin{tabular}{|c|c|c|c|}
\hline & Poorest Eaters & Class 2 & $\begin{array}{c}\text { Class } 3 \\
\text { Active, super- } \\
\text { eaters, highest } \\
\text { screen time }\end{array}$ \\
\hline \multicolumn{4}{|l|}{ Demographics (n (\%)) } \\
\hline $\mathrm{N}$ & $233(22 \%)$ & $399(37 \%)$ & $441(41 \%)$ \\
\hline \multicolumn{4}{|l|}{ Race } \\
\hline White & $198(85 \%)$ & $361(90 \%)$ & $394(89 \%)$ \\
\hline Black/AA & $15(6 \%)$ & $9(2 \%)$ & $25(6 \%)$ \\
\hline Other & $20(9)$ & $29(7 \%)$ & $22(5 \%)$ \\
\hline Hispanic origin (yes) & $19(8 \%)$ & $14(4 \%)$ & $22(5 \%)$ \\
\hline \multicolumn{4}{|l|}{ Household Income } \\
\hline$<\$ 39 \mathrm{~K}$ & $62(27 \%)$ & $82(21 \%)$ & $125(28 \%)$ \\
\hline$\$ 40-74999$ & $78(33 \%)$ & $123(31 \%)$ & $145(33 \%)$ \\
\hline$\$ 75-99999$ & $37(16 \%)$ & $76(19 \%)$ & $80(18 \%)$ \\
\hline$\$ 100-149000$ & $39(17 \%)$ & $75(19 \%)$ & $66(15 \%)$ \\
\hline$>\$ 150000$ & $17(7 \%)$ & $43(11 \%)$ & $256 \%)$ \\
\hline Sex (female) & $135(58 \%)$ & $188(47 \%)$ & $226(51 \%)$ \\
\hline Children with Obesity (BMI \% > 95) & $43(18 \%)$ & $39(10 \%)$ & $61(14 \%)$ \\
\hline $\begin{array}{l}\text { Health Behaviors (means } \pm \text { SD) } \\
\text { Physical Activity (days active at least } 60 \\
\text { minutes) }\end{array}$ & $4.94 \pm 1.74$ & $5.49 \pm 1.63$ & $5.71 \pm 1.46$ \\
\hline Screen Time (minutes/day) & $103.25 \pm 74.18$ & $43.73 \pm 29.43$ & $122.79 \pm 70.99$ \\
\hline Breakfast consumption (days/week) & $6.42 \pm 1.42$ & $6.97 \pm 0.17$ & $6.59 \pm 1.05$ \\
\hline Fruit Intake (servings/day) & $0.38 \pm 0.30$ & $1.84 \pm 0.96$ & $1.47 \pm 1.06$ \\
\hline Vegetable Intake (servings/day) & $0.39 \pm 0.28$ & $1.85 \pm 1.03$ & $1.53 \pm 1.23$ \\
\hline Fast Food Intake (times/week) & $1.20 \pm 1.00$ & $0.51 \pm 0.73$ & $1.02 \pm 0.83$ \\
\hline Sugar Sweetened Beverages (servings/day) & $0.42 \pm 0.68$ & $0.15 \pm 0.22$ & $0.59 \pm 0.88$ \\
\hline Sleep (hours per night) & $9.46 \pm 1.10$ & $9.97 \pm 0.94$ & $9.59 \pm 1.09$ \\
\hline
\end{tabular}


Table 3. Results from mediation analysis examining sleep duration as a mediator between class membership and BMI.

\begin{tabular}{|c|c|c|c|c|}
\hline Model & Variable & $\beta$ & $\begin{array}{l}95^{\text {th }} \% \\
\text { Confidence Limits }\end{array}$ & p-value \\
\hline \multicolumn{5}{|c|}{ Model 1. Examine the effect of class membership on BMI $\left(\% \mathrm{BMI}_{\mathrm{p} 95}\right)$} \\
\hline & Class 1: Poorest eaters & 4.11 & $(1.21,7.02)$ & 0.006 \\
\hline & Class 2: Healthy & & Reference & \\
\hline & $\begin{array}{l}\text { Class 3: Active, super-eaters, highest screen } \\
\text { time }\end{array}$ & 1.74 & $(-0.70,4.17)$ & 0.162 \\
\hline \multicolumn{5}{|c|}{ Model 2. Examine the effect of class membership on sleep (hours) } \\
\hline & Class 1: Poorest eaters & -0.51 & $(-0.68,-0.34)$ & $<0.001$ \\
\hline & Class 2: Healthy & & Reference & \\
\hline & $\begin{array}{l}\text { Class 3: Active, super-eaters, highest screen } \\
\text { time }\end{array}$ & -0.38 & $(-0.53,-0.24)$ & $<0.001$ \\
\hline \multicolumn{5}{|c|}{ Model 3. Examine the effects of sleep (hours) on BMI (\%BMI $\left.{ }_{p 95}\right)$ adjusting for class membership } \\
\hline & Sleep & -2.93 & $(-3.96,-1.90)$ & $<0.001$ \\
\hline & Class 1: Poorest eaters & 2.43 & $(-0.52,5.38)$ & 0.11 \\
\hline & Class 2: Healthy & & Reference & \\
\hline & $\begin{array}{l}\text { Class 3: Active, super-eaters, highest screen } \\
\text { time }\end{array}$ & 0.62 & $(-1.83,3.08)$ & 0.62 \\
\hline
\end{tabular}


